\title{
Technical nuances for surgery of insular gliomas: lessons learned
}

\author{
Roberto Rey-Dios, M.D., ${ }^{1}$ and Aaron A. Cohen-Gadol, M.D., M.Sc. ${ }^{2}$ \\ ${ }^{1}$ Department of Neurosurgery, University of Mississippi Medical Center, Jackson, Mississippi; and ${ }^{2}$ Goodman \\ Campbell Brain and Spine, Department of Neurological Surgery, Indiana University School of Medicine, \\ Indianapolis, Indiana
}

\begin{abstract}
Insular gliomas were traditionally considered a nonsurgical entity due to the high morbidity associated with resection. For the past 20 years, advances in microsurgical and brain mapping techniques have allowed neurosurgeons to resect insular gliomas with acceptable morbidity rates. Maximizing the extent of resection is nowadays the goal of surgery since this has proven to be an independent factor contributing to longer survival. Despite much progress, insular tumors remain a challenge for the neurosurgeon due to the complex anatomy of the region and technical expertise required to minimize morbidity during surgery. Herein, the authors describe the current surgical nuances, based on their experience and a literature review, that will allow the surgeon to achieve a thorough resection while ensuring patient safety. The key factors for successful surgery in the insular region include detailed knowledge of the surgical anatomy, mastery of the nuances of cortical and subcortical mapping methods, and meticulous microsurgical technique.
\end{abstract}

(http://thejns.org/doi/abs/10.3171/2012.12.FOCUS12342)

KEY WORDS $\quad$ insular glioma $\quad \bullet \quad$ awake craniotomy $\quad \bullet \quad$ cortical mapping $\quad \bullet$
microsurgical resection

$\mathrm{T}$ HE insula plays an important role in visceral sensorimotor processing; sympathetic control of cardiovascular tone; somatosensory input and pain processing; motor planning; volitional swallowing; and gustatory, auditive, vestibular, emotional, and cognitive functions, including language. ${ }^{36,44}$ Insular gliomas represent a unique category within intrinsic brain tumors in terms of their presentation and behavior. These tumors usually arise in areas of white matter adjacent to allocortex or mesocortex. The insula is a mesocortical structure (3-5 cellular layers) along with the temporal pole, caudal orbitofrontal cortex, and cingular and parahippocampal gyri. The cytoarchitecture of this region of the brain explains the growth pattern and expansion of these tumors. Thus, during the initial phases of growth, the tumor tends to be confined within the allo- and mesocortical areas, respecting the neocortical areas, central nuclei, and ventricles. ${ }^{50}$

The insular lobe and paralimbic region represent a common location for gliomas. Up to $25 \%$ of low-grade and $10 \%$ of high-grade newly diagnosed gliomas are found in this region. ${ }^{12}$ The insula is a challenging structure to reach from a surgical standpoint due to its complex anatomy and the adjacent highly eloquent cortices

Abbreviations used in this paper: $\mathrm{EMG}=$ electromyography; $\mathrm{EOR}=$ extent of resection; LLA = lateral lenticulostriate artery; $\mathrm{MCA}=$ middle cerebral artery; OS = overall survival; $\mathrm{PFS}=$ progression-free survival. and white matter tracts, as well as the vascular tree. For decades, tumors located in the insular region were considered inoperable. In 1992 Yaşargil et al..$^{53}$ described a safe transsylvian route to resect these tumors with an acceptable complication rate and proposed a classification based on the growth patterns of these tumors. A very useful classification system was recently proposed by Sanai et al. ${ }^{34}$ based on resectability and functional outcomes for insular gliomas, and it may be used to predict PFS and OS. For the past 20 years, the development and implementation of cortical and subcortical motor mapping techniques under awake and sleep conditions have improved the safety of this operation.

Although a number of review articles are available regarding the surgical management of insular gliomas, a discussion of technical nuances for handling these challenging tumors may be helpful to novice neurosurgeons who plan to evolve their technique. We will review technical considerations to minimize complications during microsurgical excision of insular gliomas. The senior author (A.C.-G.) will describe his early personal experience and lessons learned from resection of 25 insular gliomas (10 dominant and 15 nondominant tumors). The accompanying videos will further illustrate and elaborate on the following technical details.

\section{Surgical Anatomy}

The microsurgical, topographic, and vascular anat- 


\section{R. Rey-Dios and A. A. Cohen-Gadol}

omy of the insula has only recently been described in detail. ${ }^{43-45,47}$ The insular lobe is a part of the cerebral cortex covered by the opercula of the frontal, parietal, and temporal lobes. The 3D structure of the insula constitutes a pyramid, and its apex represents the most lateral and superficial point of the structure, located 9-16 $\mathrm{mm}$ from the cortical surface. ${ }^{44}$ The insular apex is located beneath the anterior sylvian point, found just inferior to the vertex of the pars triangularis of the inferior frontal gyrus. The anterior sylvian point is one of the widest portions of the fissure where the dissection can be initiated. ${ }^{32}$ The insular cortex is circumscribed by the anterior, superior, and inferior periinsular sulci. The identification of superior and inferior periinsular sulci is important from a surgical standpoint since an early exposure at their base is necessary to adequately reach the inferior and superior borders of insular tumors. The convergence of the anterior and superior periinsular sulci is known as the anterior insular point, whereas the posterior insular point is located at the intersection of the superior and inferior periinsular sulci. The sulcal-gyral anatomy of the insular cortex is detailed in Fig. 1. Two important anatomical landmarks of the insular lobe are the insular stem, which is the anterobasal portion of the insula located in the depth of the proximal sylvian fissure, and the limen insulae, located within the insular stem.

Several critical structures are located medial and deep to the insula. The extreme capsule, claustrum, external capsule, and striatum are located deep to the central portion of the insula. The fibers of the motor cortex converging into the posterior limb of the internal capsule run immediately deep to the posterior segment of the superior periinsular sulcus. The uncinate fasciculus runs medial to the anterior portion of the superior periinsular sulcus. ${ }^{43,44,47}$

The course of the MCA along the insular surface poses certain challenges during surgery. The insula receives most of its vascular supply from short perforating vessels originating from the $\mathrm{M}_{2}$ and $\mathrm{M}_{3}$ segments of the MCA. ${ }^{43,45,47}$ These short perforators, often engulfed and hidden by the superficial aspects of the tumor, can be safely coagulated and cut during subpial resection, effectively devascularizing the tumor. The $\mathrm{M}_{2}$ segments also give rise to long perforating branches that travel posteriorly and superiorly on the insula and supply the corona radiata $^{45}$ (Figs. 2 and 3). These branches must be preserved to avoid ischemic injury resulting in hemiparesis. ${ }^{25}$ The $\mathrm{M}_{1}$ segment gives origin to the LLAs as it courses under the anterior perforated substance to emerge on the limen insulae. The number of LLAs can vary from only 1 to up to 15 (Fig. 4). ${ }^{45}$ These arteries supply the basal ganglia and internal capsule, but in cases of large tumors, they can be a source of vascular supply to the insula along with the $\mathrm{M}_{2}-\mathrm{M}_{3}$ short perforating branches. Early identification of the LLAs is key to avoid ischemic injury to the medial structures. Early dissection of the proximal $\mathrm{M}_{1}$ segment allows the surgeon to follow the artery and to identify the most lateral LLAs. These LLAs represent the most medial limit of tumor resection. ${ }^{25,52,53}$

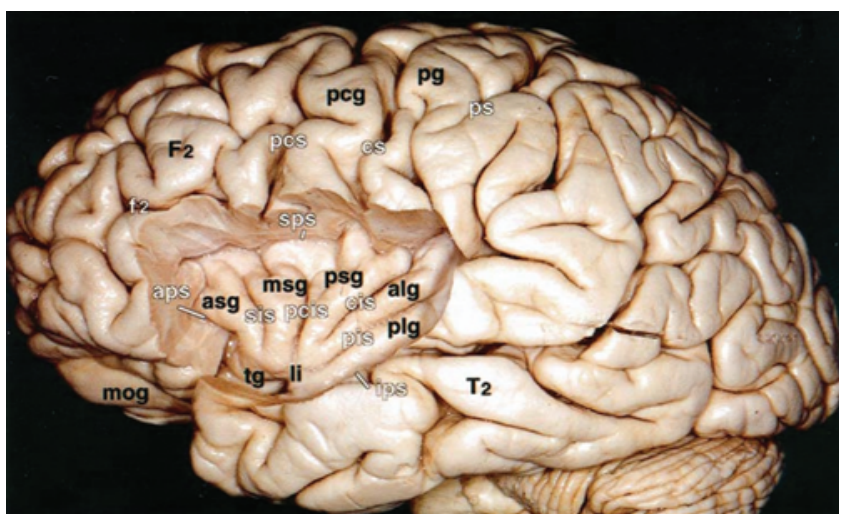

FIG. 1. Photograph of a cadaveric specimen after excision of the opercula detailing the insular cortex. Abbreviations with white letters denote sulci and fissures. alg = anterior long insular gyrus; aps = anterior periinsular sulcus; asg = anterior short insular gyrus; cis = central insular sulcus; $c s=$ central sulcus of Rolando; $F_{2}=$ middle frontal gyrus; $f_{2}=$ inferior frontal sulcus; ips = inferior periinsular sulcus; $l i=$ limen insulae; mog = medial orbital gyrus; $\mathrm{msg}=$ middle short insular gyrus; pcg = precentral gyrus; pcis = precentral insular sulcus; $p c s=$ precentral sulcus; $p g$ = postcentral gyrus; pis = postcentral insular sulcus; $p l g$ = posterior long insular gyrus; $p s$ = postcentral sulcus; $p s g$ = posterior short insular gyrus; sis = short insular sulcus; sps = superior periinsular sulcus; $\operatorname{tg}=$ transverse insular gyrus; $T_{2}=$ middle temporal gyrus. Reprinted from Türe U et al: J Neurosurg 90:720-733, 1999, with permission.

\section{Surgical Procedure}

We prefer to resect both dominant and nondominant insular tumors under awake conditions. Nondominant insular tumors may be managed under anesthesia using sleep mapping techniques to maximize the patient's comfort. We have found mapping more efficient under awake conditions, and we can continuously monitor the patient's neurological status during removal of the medial and posterior aspect of the tumor in close association with critical motor fibers. In our experience, the patient's continuous feedback has allowed us to perform more aggressive resections without increasing neurological morbidity or causing any significant patient discomfort. During preoperative planning, the patient is carefully consulted regarding the process of an awake craniotomy to make him/her comfortable during the procedure.

\section{Patient Positioning}

The patient is positioned supine on the table with the shoulder elevated on a roll and the head turned $45^{\circ}$ contralateral to the surgical side (Fig. 5). We apply some degree of head extension to facilitate access to the superior portion of the insula under the frontoparietal operculum. This positioning method allows for a better dissection of the sylvian fissure as it facilitates the opercula to separate/fall away under the action of gravity and provides a more accessible trajectory toward the most posterior portion of the insula. The details of awake craniotomy have been described elsewhere by Berger and colleagues. ${ }^{4-6}$ We will only describe our preference/modifications for insular tumors.

An uncomfortable patient will not properly cooperate, which will make mapping difficult and restrict resection. Therefore, the degree of head rotation as well as the 


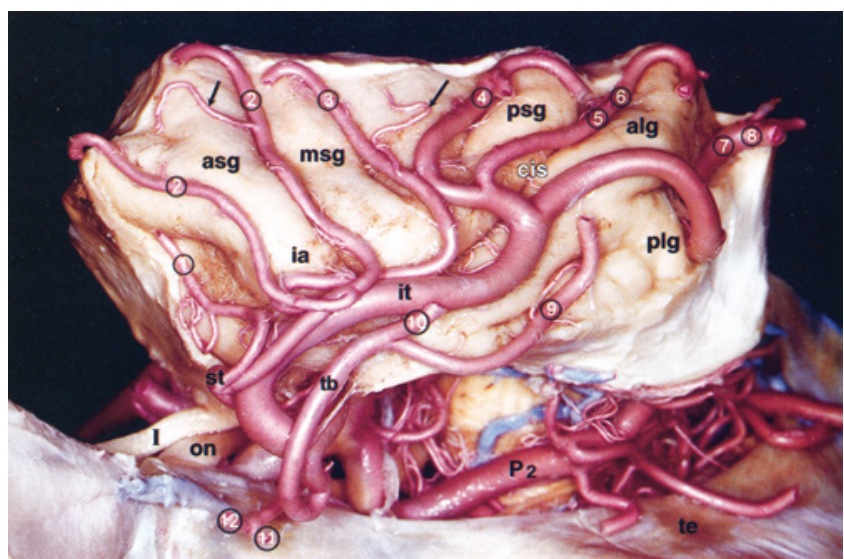

FIG. 2. Photograph of a cadaveric specimen following removal of the frontal, parietal, occipital, and temporal lobes from the periinsular sulci detailing the arteries on the insular cortex. The arteries of the insula originate from the $\mathrm{M}_{2}$ segment. The insuloopercular or long perforating arteries (arrows) supply the insula and operculum. alg = anterior long insular gyrus; asg = anterior short insular gyrus; cis = central insular sulcus; ia = insular apex; it = inferior trunk of $\mathrm{M}_{2} ; \mathrm{msg}=$ middle short insular gyrus; on = optic nerve; $P_{2}=$ ambient segment of the posterior cerebral artery; plg = posterior long insular gyrus; $p s g$ = posterior short insular gyrus; st = superior trunk of the $M_{2}$ segment; $t b=$ temporal branch of the MCA ; te = tentorium; 1 = lateral orbitofrontal artery; 2 = prefrontal artery; $3=$ precentral artery; $4=$ central artery; $5=$ anterior parietal artery; $6=$ posterior parietal artery; $7=$ angular artery; $8=$ temporooccipital artery; 9 = posterior temporal artery; $10=$ middle temporal artery; 11 = anterior temporal artery; 12 = temporal polar artery; $I=$ olfactory nerve. Reprinted from Türe et al: J Neurosurg 92:676-687, 2000, with permission.

rest of patient positioning is primarily limited by the patient's comfort. The desired degree of head rotation must be trialed before excessive narcotics and sedation have been administered. If the patient has significant discomfort with the degree of rotation that the surgeon desires, a comfortable wedge of foam can be placed under the torso on the contralateral side to compensate for the lack of neck mobility. In the awake patient, the table is bent into a "beach chair" configuration to optimize patient comfort.

Prior to placement of the skull clamp, $0.5 \%$ lidocaine with epinephrine and $0.25 \%$ bupivacaine in a $1: 1$ proportion is used to infiltrate the trajectories of the supraorbital and occipital nerves, the incision line, the root of the temporalis muscle, and pin sites. Regional scalp anesthesia will provide additional patient comfort. Whenever available, frameless stereotactic navigation should be used to localize the tumor on the surface to ensure that the scalp flap and craniotomy are large enough to generously expose the tumor and neighboring cortex to be mapped. Once resection has started, the reliability of neuronavigation diminishes due to brain shift, but for the less experienced surgeon, it can remain helpful for orientation during resection.

A "trauma flap" or question mark incision is most often used. This incision allows access to the entire length of the sylvian fissure and permits mapping of the language and sensorimotor cortex. The posterior extension of the craniotomy can be tailored based on neuronavigation data. Once the scalp flap is reflected, further
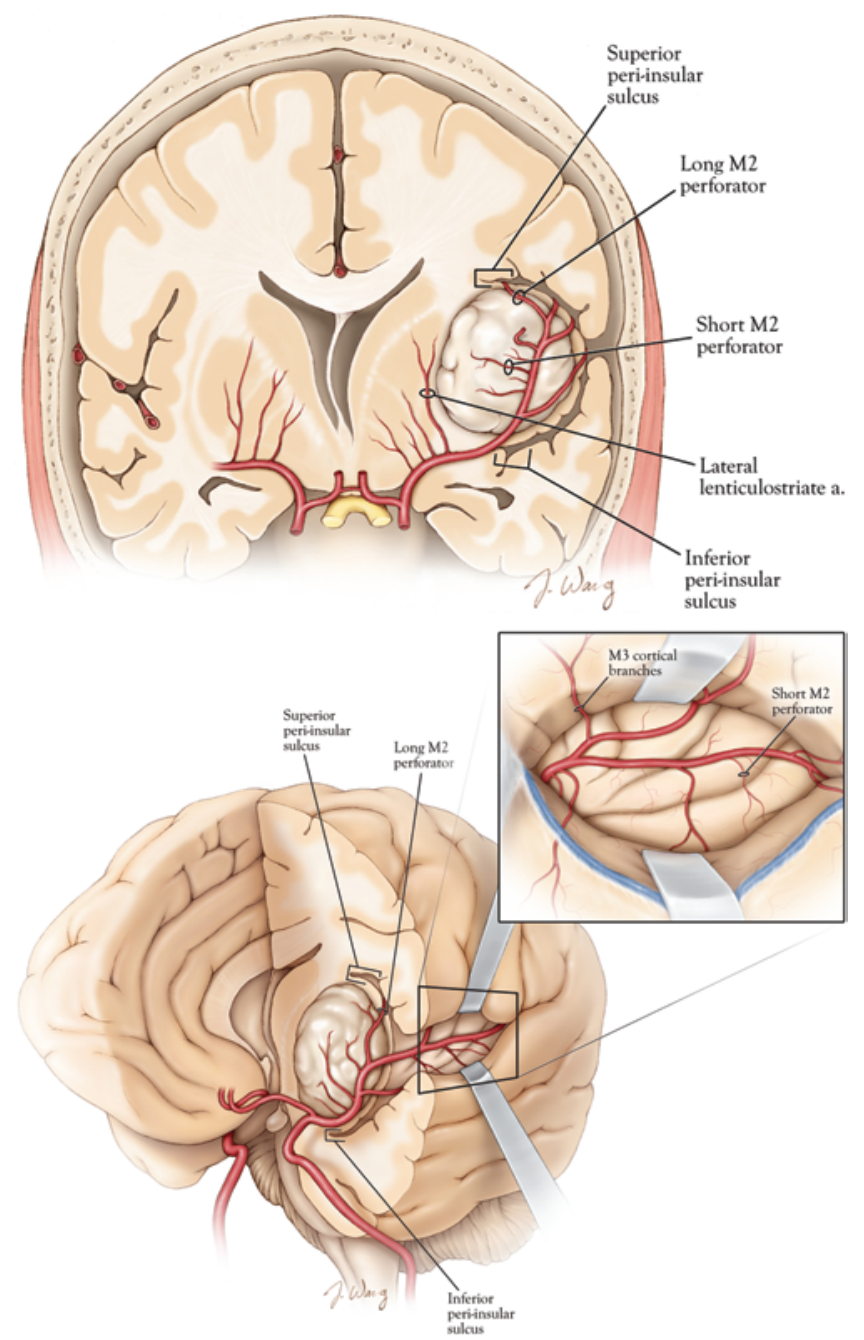

FIG. 3. Artist's depictions of the topographic and vascular anatomical relationships of insular tumors. Note how the LLAs originate from the $M_{1}$ segment and represent the medial limit of the resection. Short $\mathrm{M}_{2}$ perforators supply the tumor. Long $\mathrm{M}_{2}$ perforators may travel through the tumor but supply corona radiata and should be preserved. The superior and inferior periinsular sulci represent the superior and inferior anatomical margins of the resection. a. = artery. Printed with permission from Aaron A. Cohen-Gadol.

infiltration of the temporalis muscle is necessary. Upon elevation of the bone flap, the dura is infiltrated with $0.5 \%$ lidocaine with a very fine needle following the trajectory of the middle meningeal artery and radially along the craniotomy edge. If the patient had to be deeply sedated for craniotomy, the process of reawakening should take place after sylvian fissure dissection has been completed and the lateral portion of the insular tumor through the transsylvian route has been removed.

\section{Sylvian Fissure Dissection}

Sylvian fissure dissection provides a narrow corridor for resection of most insular tumors. In addition, MCA branches often tether the frontal lobe to the temporal lobe, limiting elevation of the frontal lobe and undermining of its operculum to remove tumor underneath 


\section{R. Rey-Dios and A. A. Cohen-Gadol}

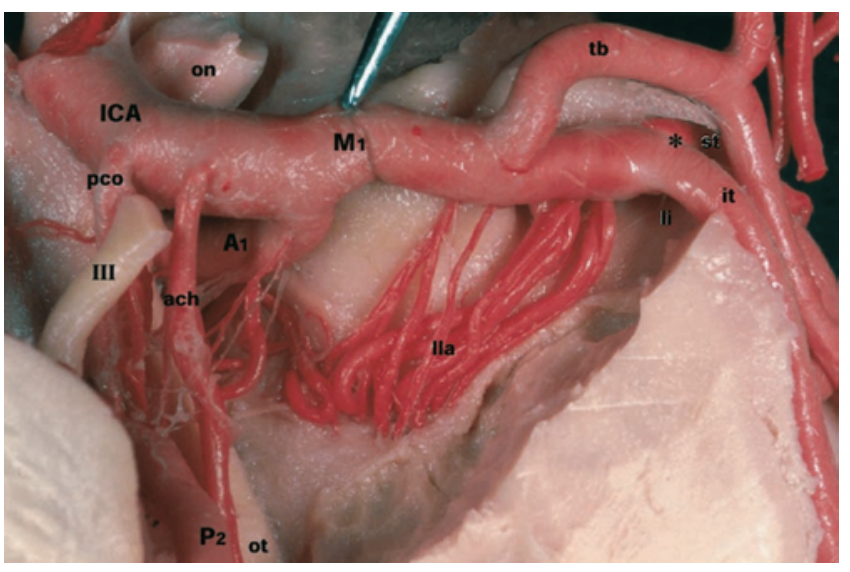

FIG. 4. Photograph of a cadaveric specimen following removal of the temporal lobe, showing an inferior view of the left MCA in the region of the anterior perforated substance. Note how the lateral lenticulostriate arteries (Ila) originate from the $M_{1}$ segment as it bifurcates into $M_{2}$ branches (asterisk). $A_{1}=$ precommunicating segment of the anterior cerebral artery; ach = anterior choroidal artery; ICA = internal carotid artery; it = inferior trunk of the $M_{2}$ segment; $l i=$ limen insulae; $M_{1}=$ sphenoidal segment of the MCA; on = optic nerve; ot = optic tract; $P_{2}$ = ambient segment of posterior cerebral artery; $\mathrm{pco}=$ posterior communicating artery; st = superior trunk of the $\mathrm{M}_{2}$ segment; tb = temporal branch of the MCA; III = oculomotor nerve. Reprinted from Türe et al: $J$ Neurosurg 92:676-687, 2000, with permission.

the frontoparietal operculum. Since these tumors often have temporal or frontal extensions, additional cortical incisions/resections within the frontal and temporal opercula are necessary to allow the surgeon enough space to maximize tumor excision. Therefore, mapping of the face area (dominant and nondominant tumors) or Broca and Wernicke areas (dominant tumors), is necessary to further guide the location of the safe cortical incision(s) in the inferior frontal and superior temporal gyri to extend the working space and angles to facilitate further tumor exposure.

Under the operating microscope and using microsurgical techniques, we apply the "inside to outside" technique to efficiently split the fissure widely for exposure of the insular cortex (please refer to Video 1 for a demonstration of this technique)..$^{51,53}$

VIDEO 1. Video clip showing the technical nuances for sylvian fissure dissection and exposure of the insula. Click here to view with Media Player. Click here to view with Quicktime.

The distal part of the fissure harboring the generous sylvian cistern is dissected open, and this dissection is extended to the depth of the fissure to identify distal MCA branches. Next, the overlying thick superficial sylvian arachnoid membranes are also disconnected. We then use this distal deep opening within the fissure and identify the distal MCA branches as a landmark to further open the fissure from "inside to outside" or deep to superficial. We avoid the common "outside to inside" technique, which is more difficult to perform due to the adherence of frontal and temporal opercula and lack of any landmarks to guide dissection of the interdigitating opercula.

After extension and completion of the sylvian fissure dissection more medially along the distal $\mathrm{M}_{1}$ segment, the

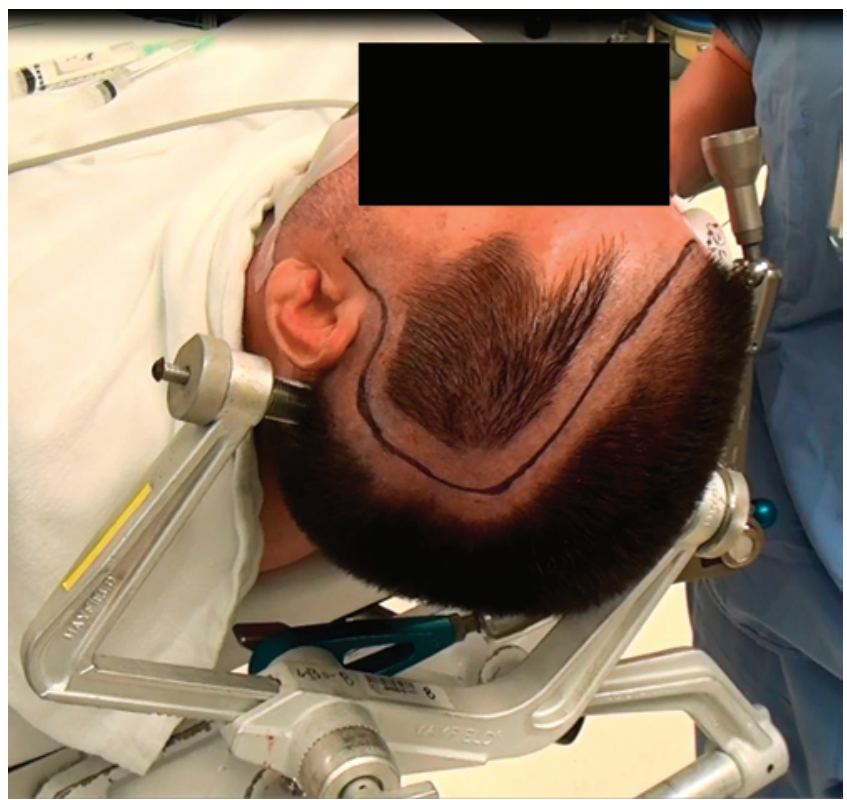

FIG. 5. Photograph of the patient under local anesthesia positioned for resection of a left insular glioma. We prefer to place the single pin of the skull clamp on the mastoid to have full access to the convexity.

temporal operculum is mobilized away from the insula. The presence of MCA branches between the temporal operculum and the insular cortex makes early mobilization of the temporal operculum easier than the frontal operculum. Occasional bridging sylvian veins are coagulated and cut. At this point, proximal fissure dissection is extended to the distal $\mathrm{M}_{1}$ segment and LLAs to decrease the required amount of retraction on the opercula.

Distal fissure dissection is often limited due to adherence of the opercula at this level; aggressive manipulation in this area will place the superior temporal gyrus at risk for injury. Gentle retraction of the frontal and temporal opercula often provides good exposure of the insular cortex. Infiltration of the insular cortex by tumor may cause bleeding during dissection of the insular cortex, and $\mathrm{M}_{2}$ short perforating branches may be sacrificed (carefully coagulated and cut, not avulsed) for hemostasis (Figs. 6 and 7). Generous exposure of the insular cortex without aggressive retraction on the opercula is paramount. The LLAs define the medial extent of the exposure and resection. They are usually displaced medially and rarely provide vascular supply to the tumor (Fig. 3). The superior and inferior periinsular sulci define the superior and inferior extent of the operative corridor, although these landmarks are often affected by tumor expansion. Exposure of the base of these sulci is critical in providing an adequate working corridor for tumor resection. The longer posteriorly and superiorly located $\mathrm{M}_{2}-\mathrm{M}_{3}$ perforators traveling toward the central lobule are strictly protected.

Some insular tumors expand the insula and may protrude through the sylvian fissure. This finding usually means significant adherence of the frontal and temporal opercula to the insular cortex, making dissection more difficult.

\section{Insular Tumor Resection}

Before the patient is awakened, we remove the lateral 


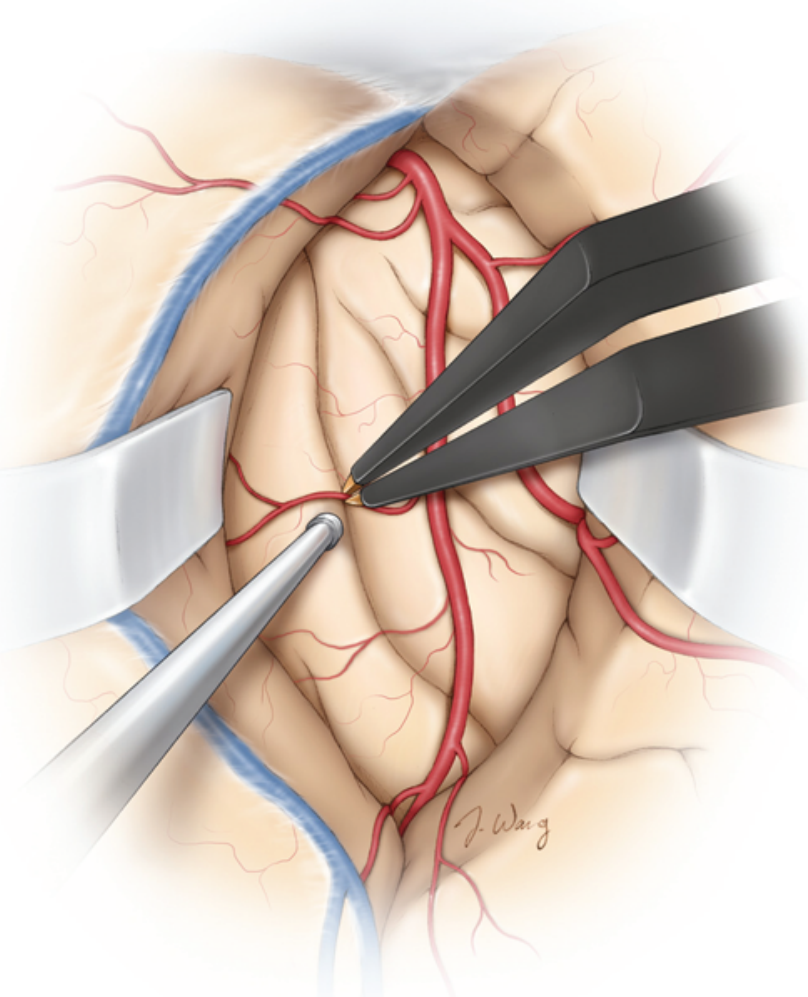

Fig. 6. The $\mathrm{M}_{2}$ perforators on the insular cortex must be thoroughly coagulated and sharply cut to avoid injury to the parent vessel by avulsion. Printed with permission from Aaron A. Cohen-Gadol.

insular portion of the tumor. To accomplish this step, the remaining perforating arteries from $\mathrm{M}_{2}$ branches leading to the insular cortex are carefully coagulated and cut. Again, avulsion of any of these perforating arteries and aggressive manipulation of the $\mathrm{M}_{2}$ arteries will place important MCA branches at risk for vasospasm and distal ischemia. We use small pieces of Gelfoam (Pharmacia and Upjohn Company) soaked in papaverine solution to cover the $\mathrm{M}_{2}$ branches to minimize their vasospasm during their mobilization and manipulation. At this point, a number of windows among and around the $\mathrm{M}_{2}$ branches are available to complete multiple insular corticotomies to provide various working angles to achieve tumor removal (Fig. 8). The $\mathrm{M}_{2}$ branches are mobilized and handled gently as Gelfoam soaked in papaverine solution is used to periodically cover them and minimize their spasm. We complete a conservative intracapsular tumor removal but avoid handling the tumor margins until later in the operation while using subcortical mapping under awake conditions (Fig. 9).

The extent of tumor removal and use of neuronavigation will determine the location of cortical incision(s) along the inferior frontal or superior temporal gyri to expand the surgeon's working zone. We avoid ultrasonic aspirators in the fear of MCA injury and use bipolar electrocautery and suction to emulsify and remove the tumor. It is important to always keep the MCA branches and LLAs in sight to avoid their inadvertent injury by ex-

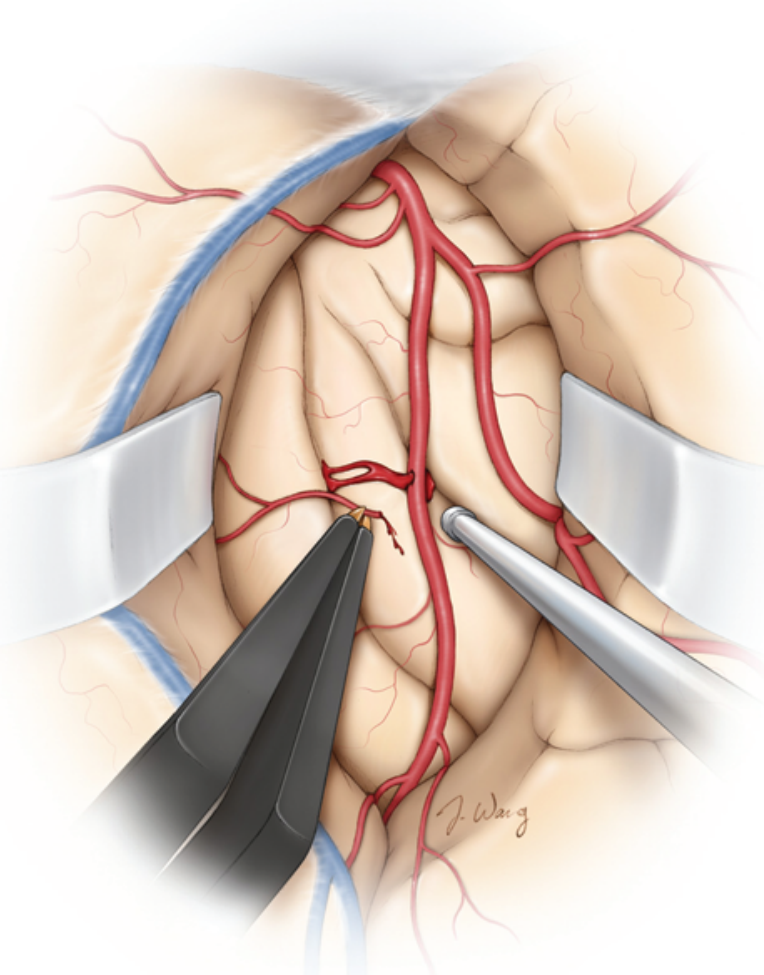

Fig. 7. Excessive manipulation of the $M_{2}$ arteries can result in avulsion of the perforating branches. Avulsion injury on the parent vessel can result in severe spasm and even occlusion, causing catastrophic ischemic injury to distal territories. Printed with permission from Aaron A. Cohen-Gadol.

cessive retraction. Meticulous hemostasis will assist with frequent identification of these structures and their safety.

\section{Intraoperative Neurophysiological Monitoring and Mapping}

Advances in brain mapping, primarily due to the work of Berger and Ojemann ${ }^{4}$ and Ojemann et al. ${ }^{31}$ account for the decrease in surgical morbidity and increase in the EOR reported in recent years. As the removal of the insular portion of the tumor is completed, the need for expansion of the working zone will often require the surgeon to map function along the inferior frontal and superior temporal gyri to expose additional tumor covered by these gyri. As mentioned previously, tumors on the nondominant side can be resected under general anesthesia with motor mapping of the face area during this stage of the operation. Tumors on the dominant side require awake speech mapping under local anesthesia to protect language function along the inferior frontal and superior temporal gyri. These mapping techniques are briefly described below.

\section{Electroencephalography and Electrocorticography}

Surface electroencephalography is recorded bilaterally by placing a pair of subdermal scalp electrodes on 


\section{R. Rey-Dios and A. A. Cohen-Gadol}

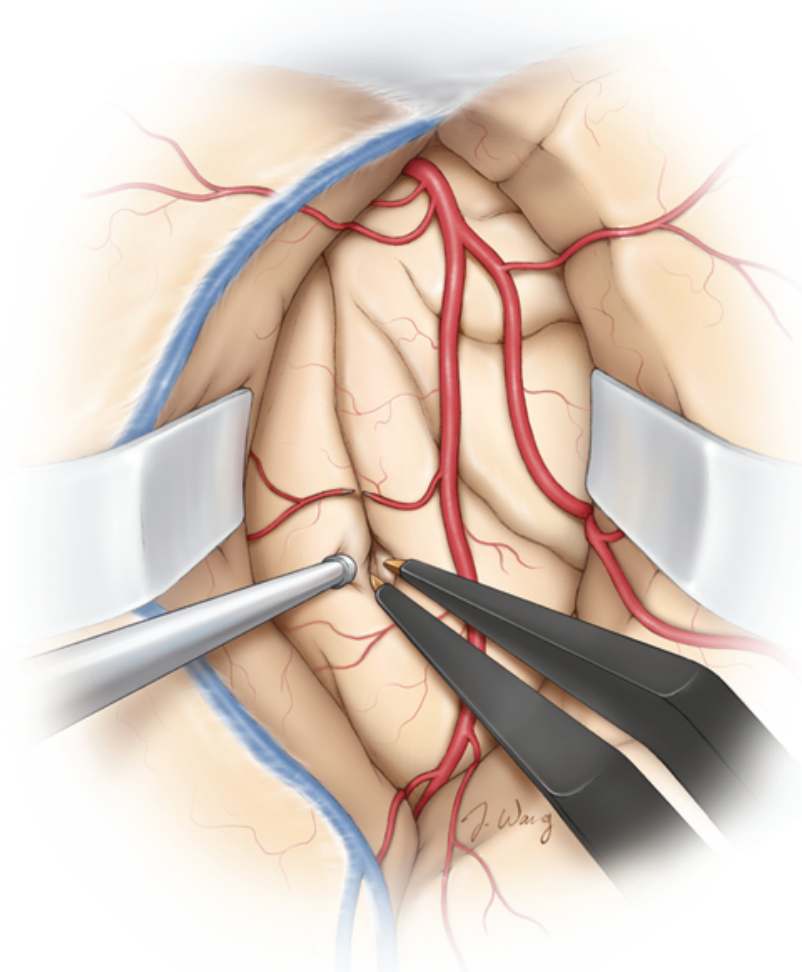

FIG. 8. Once the perforating arteries have been coagulated and divided, several pial incisions can be made on the insular cortex between the $M_{2}$ branches, thus opening several windows for subpial resection of the tumor. Printed with permission from Aaron A. Cohen-Gadol.

each side. Surface electroencephalography information is useful to assess the depth of anesthesia, thus serving as a quality control for other modalities. A subdural strip with 4-8 contact electrodes configured in a monopolar array is introduced onto the subdural space adjacent to the craniotomy opening. A single frontal electrode is used for reference. This configuration allows recording of electrocorticography activity in the cortex adjacent to the operative field to assess for afterdischarges triggered by direct cortical stimulation. ${ }^{7}$

\section{Direct Electrical Stimulation}

Direct electrical stimulation provides a detailed functional map that can be easily reproduced..$^{3,5,31}$ Cortical stimulation is performed to localize the cortical motor areas, and subcortical stimulation during resection allows for preservation of the descending subcortical white matter tracts. ${ }^{5}$

\section{Motor Mapping Under General Anesthesia}

In the anesthetized patient, motor mapping of the face area can be performed by visual inspection of the contralateral muscle groups. However, the sensitivity of the mapping greatly increases with the use of EMG..$^{54} \mathrm{In}$ this situation, multiple muscle groups, including the face (and tongue and pharynx, if so desired), can be monitored with needle electrodes. An Ojemann Cortical Stimulator (Integra LifeSciences) with 1 -mm electrode tips $5 \mathrm{~mm}$

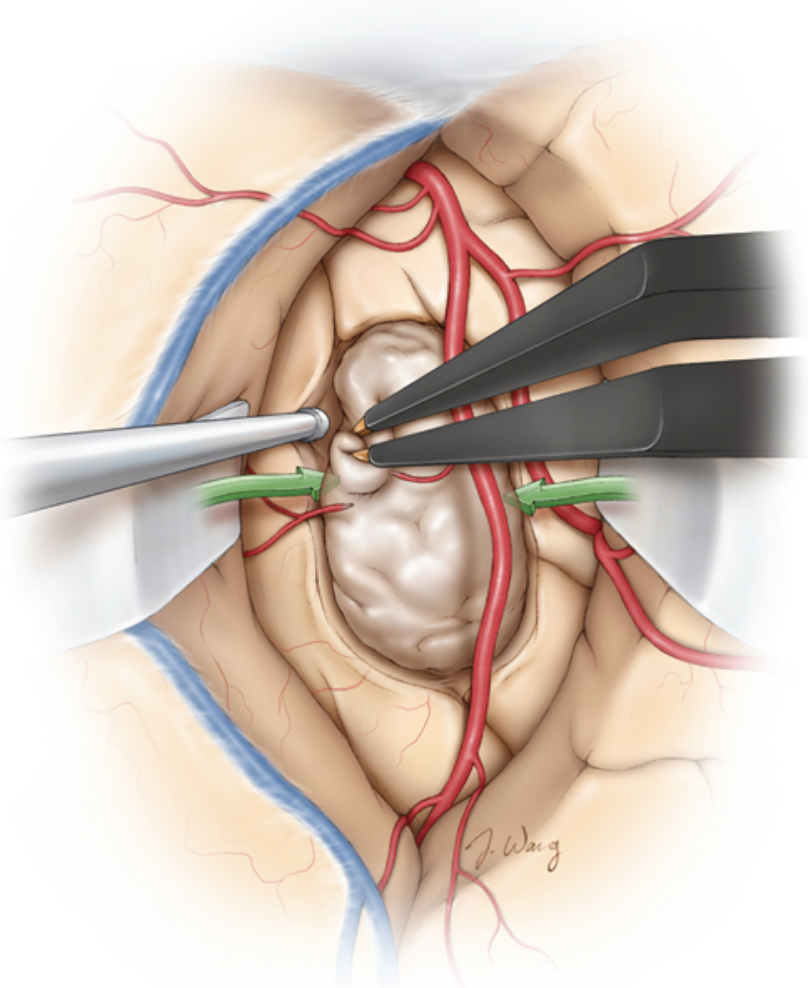

Fig. 9. Intracapsular resection of the bulk of the tumor is performed as an initial step in the resection. The intracapsular margins of the tumor are defined during dissection circumferentially, and the core of the tumor is removed using bipolar coagulation and suction. Printed with permission from Aaron A. Cohen-Gadol.

apart delivering biphasic square wave pulses is used for both cortical and subcortical mapping. Initial identification of the central sulcus and motor strip can be done by anatomical landmarks, neuronavigation, or by somatosensory motor potential phase reversal (please see below). This last modality can be very useful and time saving, especially during pediatric cases when the anatomy might be difficult to interpret. In children younger than 5 years of age, direct stimulation might not be feasible or elicit a response. ${ }^{3,21}$

In the anesthetized patient, the starting current is usually $4 \mathrm{~mA}$ and is increased at $2-\mathrm{mA}$ intervals until an EMG or a motor response is visually identified in the face. The highest stimulation current usually does not exceed $18 \mathrm{~mA}$. The bipolar probe is applied to the cortex for $1-2$ seconds. If a positive response is achieved, the area is marked with a numbered or lettered ticket/tag. The areas immediately adjacent to the one that elicited the movement should also be stimulated and mapped. The spread of the bipolar probe is minimal, only $2-3 \mathrm{~mm} .{ }^{24}$ Electrical activity in the surrounding cortex (afterdischarges) is continuously monitored by means of a subdural electrode strip array. If afterdischarges are detected after stimulation, the mapping process should stop for a few seconds until the brain returns to baseline activity. The amplitude used to continue mapping should be $1-0.5 \mathrm{~mA}$ lower than the one that elicited afterdischarges. Only the responses 
obtained in absence of afterdischarges are considered to be reliable for mapping. If electrical or clinical seizure activity is detected, cold Ringer solution should be readily available to irrigate the cortex. ${ }^{35}$ It is important to avoid displacing the tags while irrigating the cortex. If unsuccessful, short-acting drugs (propofol and midazolam) may be rarely required to prevent generalization of the seizure.

\section{Somatosensory Evoked Potential Phase Reversal}

This technique can help the surgeon to quickly identify the central sulcus, and therefore it expedites the mapping process during sleep mapping. A subdural strip with 4-8 electrode contacts is placed across the presumed central sulcus identified based on anatomical landmarks. The median nerve on the contralateral side is stimulated, and the somatosensory evoked potentials are recorded from the strip. Electrodes located on the sensory cortex will display a negative-positive waveform configuration, whereas the ones located on the motor cortex will display a positive-negative morphology. The 2 electrodes that display a mirror configuration are located on either side of the central sulcus. The amplitude of the waveform is usually largest on the hand area upon stimulation of the median nerve. The same technique can be used by stimulation of the tibial nerve and localization in the areas close to the midline. ${ }^{21,22,48,49}$

\section{Electromyography}

Continuous multichannel EMG recording is used in the anesthetized patient to improve the sensitivity of motor mapping. Several agonist and antagonist proximal and distal muscle groups are monitored in upper and lower extremities. Face electrodes are also placed. During direct cortical stimulation, the contralateral side of the body and face is visually monitored for movement. Recording of EMG activity can detect subclinical muscle activity and facilitate mapping at lower stimulation amplitudes. ${ }^{7,54}$

\section{Motor Evoked Potentials}

Continuous monitoring of motor evoked potentials represents another neurophysiological tool to monitor the integrity of motor cortex and subcortical motor tracts during tumor resection. This monitoring modality has been modified for use under general anesthesia, ${ }^{42}$ as it was initially used during vascular neurosurgical procedures to monitor for signs of ischemia. ${ }^{41}$ This modality has been found to be sensitive for detection of imminent injury to the motor cortex and subcortical motor pathways during tumor resection. ${ }^{8,29} \mathrm{~A}$ strip electrode containing 4-8 electrodes is placed over the precentral gyrus. A train of 2-4 pulses (anodal current close to motor threshold, duration $0.3-0.5 \mathrm{msec}$, interstimulus interval $4 \mathrm{msec}$ ) is usually sufficient to elicit muscle activity. ${ }^{40,42}$ The multichannel EMG can be recorded via needle electrodes in the anesthetized patient or surface electrodes in awake cases. This monitoring method provides real-time information on the integrity of motor pathways during resection when direct electrical stimulation is not being performed. This method has also been found to be sensitive to impending brain ischemia, which represents a potential major complica- tion during insular tumor resection, when direct damage or vasospasm of en passage arteries during the resection can result in distal territory strokes with catastrophic consequences. ${ }^{30}$

\section{Motor Mapping in the Awake Patient}

The overall setup for motor mapping in the awake patient is similar to the one used for the asleep patient. Motor response via direct electrical stimulation is readily observed in the awake patient. The patient is interrogated about any involuntary pharyngeal or tongue movement. In the awake patient, the intensity of current needed to obtain a motor response is usually lower. Thus, the starting intensity is $2 \mathrm{~mA}$ and is increased at intervals of 1 $\mathrm{mA}$ until motor responses or afterdischarges are detected. In awake patients, the highest intensity needed is usually $8-12 \mathrm{~mA}$. Once movement is perceived, the entire exposed cortical area should be stimulated every $5 \mathrm{~mm}^{2}$ (when using a probe with tips spaced $5 \mathrm{~mm}$ ). Every site should be stimulated at least 3 times for confirmation, but not consecutively. In cooperative awake patients, negative motor testing can be performed. ${ }^{28}$ For this purpose, the patient is instructed to perform a repetitive task. Stimulation of the associative motor areas inhibits the corresponding movement. ${ }^{13}$

\section{Language Mapping}

Wide exposures and large craniotomies are required to accomplish this task. Mapping performed through a limited exposure can be risky and should be performed in experienced hands. In this case, mapping relies on negative stimulation rather than the location of positive stimulation areas. ${ }^{33}$

In the awake patient, stimulation is performed starting at a lower current (1.5-2 mA). The highest amplitude needed rarely exceeds $8-12 \mathrm{~mA}$. The principles for cortical language mapping were established by the seminal work by Ojemann and colleagues. ${ }^{31}$ A practical approach is to find the motor areas corresponding to the face and hand. The highest current that can elicit a motor response without afterdischarges is used to map speech. The patient is asked to start counting from 1 to 10 repeatedly. The Broca area should be adjacent to the face and hand areas. For speech mapping, the current is applied for 4 seconds at a time. Stimulation of the Broca area will cause speech arrest. This phenomenon must be differentiated from dysarthria induced by motor activity in the muscles of the face, tongue, or pharynx. Once the motor speech area is localized, a sterile ticket tags the area. ${ }^{23}$ This area can be diffuse and should be thoroughly mapped.

The next step is to map the exposed areas of the superior temporal gyrus. In our experience, corticotomies along the anterior regions of the inferior frontal and superior temporal gyri provide an adequate operative corridor to remove tumors located beyond the boundaries of insula accessible through the transsylvian route.

The same current intensity that caused speech arrest in the Broca area is applied to the superior temporal gyrus and is sequentially increased in intensity $1 \mathrm{~mA}$ at a time until a brief afterdischarge from the temporal lobe is noted. The current is set for mapping $1 \mathrm{~mA}$ below the 


\section{R. Rey-Dios and A. A. Cohen-Gadol}

afterdischarge threshold. In this region, the goal of stimulation is to cause disruption of naming. Stimulation at the previously determined intensity is applied for 4 seconds on each location. The patient is shown slides that contain a brief sentence such as "This is a..." and a drawing of a common object or action. The patient reads the sentence that precedes the picture to ensure that no disturbances are causing speech arrest while the slide is being presented. ${ }^{18}$ The slides are presented every 4 seconds, and the stimulation is applied at the time the slide is shown. Two slides are presented between stimulations to ensure that there is no underlying seizure that could be disturbing normal language function. Depending on the temporal extension of the insular tumor, the superior and inferior temporal gyri may need to be stimulated. If a site where stimulation causes naming disturbance is found, this area should be stimulated 3 nonconsecutive times for confirmation. The disturbance in naming should occur during at least 2 of 3 stimulations, and naming should normalize immediately after the stimulation is stopped in order for the area to be considered essential for speech. ${ }^{16,31}$ It is recommended to keep the corticotomy/resection margin at minimum $7 \mathrm{~mm}$ away from the eloquent area to minimize the incidence of postoperative language dysfunction. ${ }^{31}$ However, other studies have demonstrated that if cortical and subcortical stimulation is continuously used during resection, a safety margin might not be always necessary. ${ }^{20}$ The incidence of transient deficits, especially speech disturbance, has been found to be higher when safety margins are not respected, but most patients recover in a 3-month period. ${ }^{18}$

\section{Subcortical Mapping}

A growing body of evidence suggests that subcortical mapping can be a valuable tool during resection of gliomas to prevent morbidity. ${ }^{13,26}$ Subcortical motor mapping is performed at the same current intensity or slightly higher than the one for a cortical response. This method allows for identification of white matter motor tracts during resection. Along with continuous MEP monitoring, this technique affords maximal tumor resection with decreased morbidity. Subcortical mapping is performed in a repetitive manner, such that resection (3-mm-thick layers) is followed by stimulation, and once a motor response is found, resection is stopped. ${ }^{4,5,7}$ Subcortical mapping is paramount for superior, medial, and posterior margins of resection in cases of insular gliomas, since such tumors can be medial enough to significantly displace or invade descending motor tracts. ${ }^{38}$

Subcortical mapping allows the surgeon to perform a more thorough resection safely, since neuronavigation is not reliable at this point of the operation when the brain has shifted due to initial resection and egress of CSF. Subcortical language mapping is currently performed at some institutions. For insular tumors, mapping of the deep portion of the opercula and the insular cortex has been found to be important as significant language functions have been found in these locations. This technique has recently provided important information about the subcortical organization of language,,$^{1,16}$ but its practical applications are not yet well understood.

\section{Further Steps in Resection}

After initial removal of the tumor through the transsylvian route and mapping of the inferior frontal and superior temporal gyri, additional corticotomies in the negatively mapped areas of the opercula allow for further exposure of the tumor. Tumor removal continues with the aid of subcortical mapping. The nutmeg appearance of the striatum should be familiar to the surgeon. The LLAs are often encountered before the striatum and strictly define the more medial and anterior EOR (Fig. 10). The suction apparatus can easily injure these perforators. Any bleeding in this medial region should be controlled with gentle pressure of a piece of Gelfoam soaked in thrombin solution, and bipolar coagulation is avoided. Identifying the depth of resection can be very challenging, and the distal LLAs provide a reasonable landmark. Neuronavigation may provide additional information at this juncture as the medial structures are least affected by brain shift. A highly magnified and well-illuminated surgical field through the use of the operating microscope and surgical experience also provides the surgeon with changes in consistency, color, and texture, which lead to clues about reaching the margins of the tumor in relation to normal white matter.

The aforementioned corticotomies provide an ample amount of space to remove frontal and temporal extensions of gliomas. In Video 2, resection resumed with a temporal lobectomy due to extensive temporal reach of the tumor.

VIDEO 2. Video clip showing the technical nuances for resection of an insular glioma. Click here to view with Media Player. Click here to view with Quicktime.

We then completed a lateral insular tumor resection. Finally, mapping of the face area allowed us to extend tumor resection into the posterior frontal region.

Removing the posterior extension of the tumor into the internal capsule is one of the most challenging portions of the operation and should be performed patiently during the last stages with the use of subcortical mapping.

\section{Discussion}

Insular gliomas have specific histology and natural history, are most often low grade, ${ }^{12}$ and present after a prolonged phase of growth. They are typically large at the time of presentation and frequently cause epilepsy as their only initial symptom. . $^{10,14,34}$

Resection of insular tumors was not considered a reasonable option in the past due to the high morbidity inherent to the operation. The publication of Yaşargil's experience ${ }^{53}$ with these tumors prompted neurosurgeons to revisit the surgical option. Since the publication of that work in $1992,,^{53}$ several studies have shown a positive impact of tumor resection on PFS and OS with acceptable surgical morbidity rates for both low-grade (WHO Grade I and II) $2,9,10,15,17,19,39,46,55$ and high-grade (WHO Grade III and IV) tumors. $27,34,37,46,55$ Despite the lack of Class I evidence, data from several recent studies suggest that EOR is one of the main prognostic factors in OS, PFS, and ma- 


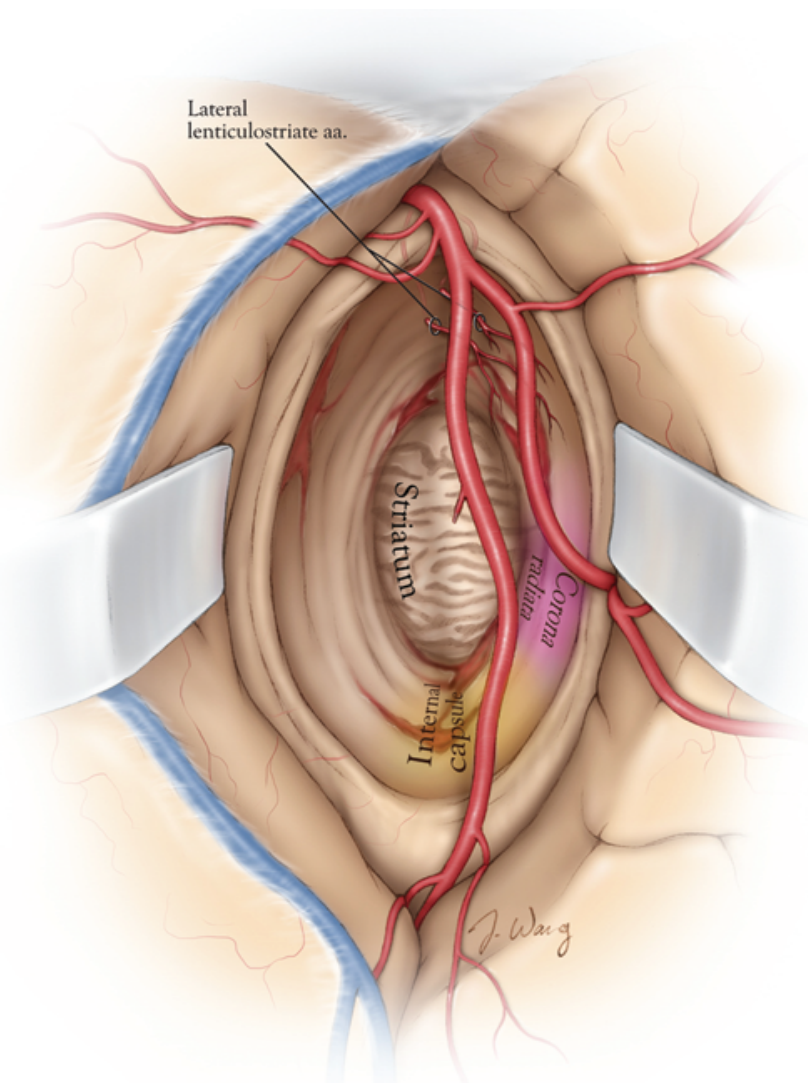

FIG. 10. View of the tumor cavity following resection depicted through the transsylvian approach for tumors confined to the insula. The bottom of the cavity is the medial limit of the resection. The LLAs and the striatum (note the characteristic nutmeg appearance) are the 2 anatomical landmarks for the medial margin of the resection. The corona radiata and the posterior limb of the internal capsule are located in the posterosuperior margin of the cavity. Further resection in this direction requires subcortical mapping to avoid injury to these white matter tracts. aa. = arteries. Printed with permission from Aaron A. Cohen-Gadol.

lignant transformation. In 2010, Sanai et al. ${ }^{34}$ published the results of resection in 115 insular gliomas. This series included 70 low-grade (WHO Grade II only; Grade I was excluded) and 45 high-grade (WHO III and IV) tumors. For low-grade gliomas, the 5-year OS increased by $16 \%$ for patients with an EOR $\geq 90 \%$. For high-grade gliomas, a $16 \%$ difference in the 2-year OS was also noted for patients with an EOR $\geq 90 \%$. Most importantly, EOR provided a significant impact in delaying malignant progression. The 5-year malignant PFS increased by $17 \%$ for patients with an EOR $\geq 90 \%$.

Another recent large surgical series of insular gliomas was published by Skrap et al. ${ }^{39}$ in 2012 and demonstrated EOR to be an independent prognostic factor for OS and PFS. This series included 66 patients with nonenhancing insular gliomas (53 WHO Grade II and $13 \mathrm{WHO}$ Grade III). The 5-year OS rate was $92 \%$ for patients with $>90 \%$ EOR and $82 \%$ for patients with EOR between $70 \%$ and $90 \%$. Patients with an EOR $<70 \%$ had a 5-year OS rate of $57 \%$.

Based on these data, maximal tumor resection, while minimizing neurological deficits, should be the goal in insular glioma surgery. Furthermore, the accumulated experience with resection of these tumors has demonstrated a significant plasticity within the region that allows most patients to compensate over time after aggressive insular resections as long as essential language and motor areas identified by stimulation during surgery are preserved. ${ }^{11,19}$

In addition to the impact on survival and malignant transformation, tumor resection has also proven to improve quality of life in patients who present with intractable epilepsy, ${ }^{10,14,37,53,55}$ a very common occurrence in these tumors.

\section{Complication Avoidance}

Ischemic injury as a result of damage to the lenticulostriate arteries or MCA branches is one of the main causes of surgical morbidity associated with insular tumor resection. To avoid these complications, a thorough knowledge of the regional anatomy, meticulous microsurgical technique, hemostasis, and gentle handling of the vasculature are crucial. The transsylvian approach requires the surgeon to be comfortable with microsurgical techniques to dissect the fissure. Insular tumors often extend into the white matter covered by frontal and temporal opercula. Initial transsylvian resection and subsequent extension of the opercular operative corridor allows for comfortable access to the insula and subsequent subpial resection with protection of the vasculature. In tumors limited to the insular lobe, we have routinely used the transsylvian approach without any significant complication.

Injury to the descending motor fibers can be another source of morbidity and should be avoided with careful identification of distal LLAs more anteriorly and subcortical mapping at the level of the internal capsule more posteriorly along the resection cavity. The long $\mathrm{M}_{2}$ perforating arteries leading to the corona radiata and central lobule should be also preserved on the surface of the insula and not coagulated inadvertently in case of excessive bleeding from the tumor.

Another common cause of morbidity after the transsylvian approach is excessive retraction on the opercular areas, which can result in damage to the Broca area, the horizontal fibers of the arcuate fasciculus near the superior periinsular sulcus, or the fibers of the uncinate fasciculus near the inferior periinsular sulcus. Damage to any of these structures can result in different degrees of speech disturbance or memory impairment, especially on the dominant side. ${ }^{18}$ To prevent this type of injury, we discourage the use of fixed retractors, and we encourage the use of a "dynamic retraction" technique, using only the suction and bipolar instruments to apply retraction to different areas while constantly shifting the pressure points (Video 2). A thorough understanding of the mapping technique becomes even more critical when limited exposures are performed, and the surgeon must rely on "negative stimulation" without identification of unexposed "positive" eloquent areas.

\section{Conclusions}

Surgical treatment has become a reasonable option 


\section{R. Rey-Dios and A. A. Cohen-Gadol}

for low-grade insular tumors. Resection of these tumors is feasible, relatively safe, and can have a significant impact on patient outcomes. The key elements of success in this surgery are 1) a thorough anatomical knowledge of the region complemented with rational use of navigation technology; 2) a thorough understanding of neurophysiological monitoring and, most importantly, cortical and subcortical mapping techniques; and 3) impeccable surgical and microsurgical technique, including gentle handling of the vasculature and normal tissues, avoidance of excessive retraction, and use of subpial resection technique. Adherence to these principles will minimize surgical morbidity and allow for a greater EOR, potentially leading to improved patient survival.

\section{Disclosure}

The authors report no conflict of interest concerning the materials or methods used in this study or the findings specified in this paper.

Author contributions to the study and manuscript preparation include the following. Conception and design: both authors Acquisition of data: both authors. Analysis and interpretation of data: both authors. Drafting the article: both authors. Critically revising the article: both authors. Reviewed submitted version of manuscript: both authors. Study supervision: Cohen-Gadol.

\section{References}

1. Bello L, Gallucci M, Fava M, Carrabba G, Giussani C, Acerbi $\mathrm{F}$, et al: Intraoperative subcortical language tract mapping guides surgical removal of gliomas involving speech areas. Neurosurgery 60:67-82, 2007

2. Berger MS, Deliganis AV, Dobbins J, Keles GE: The effect of extent of resection on recurrence in patients with low grade cerebral hemisphere gliomas. Cancer 74:1784-1791, 1994

3. Berger MS, Kincaid J, Ojemann GA, Lettich E: Brain mapping techniques to maximize resection, safety, and seizure control in children with brain tumors. Neurosurgery 25:786792, 1989

4. Berger MS, Ojemann GA: Intraoperative brain mapping techniques in neuro-oncology. Stereotact Funct Neurosurg 58: 153-161, 1992

5. Berger MS, Ojemann GA, Lettich E: Neurophysiological monitoring during astrocytoma surgery. Neurosurg Clin $\mathbf{N}$ Am 1:65-80, 1990

6. Berger MS, Rostomily RC: Low grade gliomas: functional mapping resection strategies, extent of resection, and outcome. J Neurooncol 34:85-101, 1997

7. Bertani G, Fava E, Casaceli G, Carrabba G, Casarotti A, Papagno $\mathrm{C}$, et al: Intraoperative mapping and monitoring of brain functions for the resection of low-grade gliomas: technical considerations. Neurosurg Focus 27(4):E4, 2009

8. Cedzich C, Taniguchi M, Schäfer S, Schramm J: Somatosensory evoked potential phase reversal and direct motor cortex stimulation during surgery in and around the central region. Neurosurgery 38:962-970, 1996

9. Chang EF, Clark A, Smith JS, Polley MY, Chang SM, Barbaro NM, et al: Functional mapping-guided resection of low-grade gliomas in eloquent areas of the brain: improvement of longterm survival. Clinical article. J Neurosurg 114:566-573, 2011

10. Duffau H: A personal consecutive series of surgically treated 51 cases of insular WHO Grade II glioma: advances and limitations. Clinical article. J Neurosurg 110:696-708, 2009

11. Duffau H, Bauchet L, Lehéricy S, Capelle L: Functional com- pensation of the left dominant insula for language. Neuroreport 12:2159-2163, 2001

12. Duffau H, Capelle L: Preferential brain locations of low-grade gliomas. Cancer 100:2622-2626, 2004

13. Duffau H, Capelle L, Denvil D, Sichez N, Gatignol P, Taillandier L, et al: Usefulness of intraoperative electrical subcortical mapping during surgery for low-grade gliomas located within eloquent brain regions: functional results in a consecutive series of 103 patients. J Neurosurg 98:764-778, 2003

14. Duffau H, Capelle L, Lopes M, Bitar A, Sichez JP, van Effenterre R: Medically intractable epilepsy from insular low-grade gliomas: improvement after an extended lesionectomy. Acta Neurochir (Wien) 144:563-573, 2002

15. Duffau H, Capelle L, Lopes M, Faillot T, Sichez JP, Fohanno $\mathrm{D}$ : The insular lobe: physiopathological and surgical considerations. Neurosurgery 47:801-811, 2000

16. Duffau H, Gatignol P, Mandonnet E, Peruzzi P, TzourioMazoyer N, Capelle L: New insights into the anatomo-functional connectivity of the semantic system: a study using cortico-subcortical electrostimulations. Brain 128:797-810, 2005

17. Duffau H, Moritz-Gasser S, Gatignol P: Functional outcome after language mapping for insular World Health Organization Grade II gliomas in the dominant hemisphere: experience with 24 patients. Neurosurg Focus 27(2):E7, 2009

18. Duffau H, Peggy Gatignol ST, Mandonnet E, Capelle L, Taillandier L: Intraoperative subcortical stimulation mapping of language pathways in a consecutive series of 115 patients with Grade II glioma in the left dominant hemisphere. J Neurosurg 109:461-471, 2008

19. Duffau H, Taillandier L, Gatignol P, Capelle L: The insular lobe and brain plasticity: lessons from tumor surgery. Clin Neurol Neurosurg 108:543-548, 2006

20. Gil-Robles S, Duffau H: Surgical management of World Health Organization Grade II gliomas in eloquent areas: the necessity of preserving a margin around functional structures. Neurosurg Focus 28(2):E8, 2010

21. Goldring S, Gregorie EM: Surgical management of epilepsy using epidural recordings to localize the seizure focus. Review of 100 cases. J Neurosurg 60:457-466, 1984

22. Gregorie EM, Goldring S: Localization of function in the excision of lesions from the sensorimotor region. J Neurosurg 61:1047-1054, 1984

23. Haglund MM, Berger MS, Shamseldin M, Lettich E, Ojemann GA: Cortical localization of temporal lobe language sites in patients with gliomas. Neurosurgery 34:567-576, 1994

24. Haglund MM, Ojemann GA, Blasdel GG: Optical imaging of bipolar cortical stimulation. J Neurosurg 78:785-793, 1993

25. Hentschel SJ, Lang FF: Surgical resection of intrinsic insular tumors. Neurosurgery 57 (1 Suppl):176-183, 2005

26. Keles GE, Lundin DA, Lamborn KR, Chang EF, Ojemann G, Berger MS: Intraoperative subcortical stimulation mapping for hemispherical perirolandic gliomas located within or adjacent to the descending motor pathways: evaluation of morbidity and assessment of functional outcome in 294 patients. $\mathbf{J}$ Neurosurg 100:369-375, 2004

27. Lang FF, Olansen NE, DeMonte F, Gokaslan ZL, Holland EC, Kalhorn C, et al: Surgical resection of intrinsic insular tumors: complication avoidance. J Neurosurg 95:638-650, 2001

28. Lüders HO, Dinner DS, Morris HH, Wyllie E, Comair YG: Cortical electrical stimulation in humans. The negative motor areas. Adv Neurol 67:115-129, 1995

29. Neuloh G, Pechstein U, Cedzich C, Schramm J: Motor evoked potential monitoring with supratentorial surgery. Neurosurgery 54:1061-1072, 2004

30. Neuloh G, Pechstein U, Schramm J: Motor tract monitoring during insular glioma surgery. J Neurosurg 106:582-592, 2007 
31. Ojemann G, Ojemann J, Lettich E, Berger M: Cortical language localization in left, dominant hemisphere. An electrical stimulation mapping investigation in 117 patients. J Neurosurg 71:316-326, 1989

32. Ribas GC, Ribas EC, Rodrigues CJ: The anterior sylvian point and the suprasylvian operculum. Neurosurg Focus 18(6B): E2, 2005

33. Sanai N, Berger MS: Intraoperative stimulation techniques for functional pathway preservation and glioma resection. Neurosurg Focus 28(2):E1, 2010

34. Sanai N, Polley M-Y, Berger MS: Insular glioma resection: assessment of patient morbidity, survival, and tumor progression. Clinical article. J Neurosurg 112:1-9, 2010

35. Sartorius CJ, Berger MS: Rapid termination of intraoperative stimulation-evoked seizures with application of cold Ringer's lactate to the cortex. Technical note. J Neurosurg 88:349351,1998

36. Shelley BP, Trimble MR: The insular lobe of Reil-its anatamico-functional, behavioural and neuropsychiatric attributes in humans-a review. World J Biol Psychiatry 5:176-200, 2004

37. Simon M, Neuloh G, von Lehe M, Meyer B, Schramm J: Insular gliomas: the case for surgical management. Clinical article. J Neurosurg 110:685-695, 2009

38. Skirboll SS, Ojemann GA, Berger MS, Lettich E, Winn HR: Functional cortex and subcortical white matter located within gliomas. Neurosurgery 38:678-685, 1996

39. Skrap M, Mondani M, Tomasino B, Weis L, Budai R, Pauletto $\mathrm{G}$, et al: Surgery of insular nonenhancing gliomas: volumetric analysis of tumoral resection, clinical outcome, and survival in a consecutive series of 66 cases. Neurosurgery 70:10811094, 2012

40. Szelényi A, Bello L, Duffau H, Fava E, Feigl GC, Galanda M, et al: Intraoperative electrical stimulation in awake craniotomy: methodological aspects of current practice. Neurosurg Focus 28(2):E7, 2010

41. Szelényi A, Langer D, Kothbauer K, De Camargo AB, Flamm ES, Deletis V: Monitoring of muscle motor evoked potentials during cerebral aneurysm surgery: intraoperative changes and postoperative outcome. J Neurosurg 105:675-681, 2006

42. Taniguchi M, Cedzich C, Schramm J: Modification of cortical stimulation for motor evoked potentials under general anesthesia: technical description. Neurosurgery 32:219-226, 1993

43. Tanriover N, Rhoton AL Jr, Kawashima M, Ulm AJ, Yasuda A: Microsurgical anatomy of the insula and the sylvian fissure. J Neurosurg 100:891-922, 2004

44. Türe U, Yaşargil DC, Al-Mefty O, Yaşargil MG: Topographic anatomy of the insular region. J Neurosurg 90:720-733, 1999

45. Türe U, Yaşargil MG, Al-Mefty O, Yaşargil DC: Arteries of the insula. J Neurosurg 92:676-687, 2000

46. Vanaclocha V, Sáiz-Sapena N, García-Casasola C: Surgical treatment of insular gliomas. Acta Neurochir 139:11261135,1997

47. Varnavas GG, Grand W: The insular cortex: morphological and vascular anatomic characteristics. Neurosurgery 44:127138,1999

48. Wood CC, Spencer DD, Allison T, McCarthy G, Williamson PD, Goff WR: Localization of human sensorimotor cortex during surgery by cortical surface recording of somatosensory evoked potentials. J Neurosurg 68:99-111, 1988

49. Woolsey CN, Erickson TC, Gilson WE: Localization in somatic sensory and motor areas of human cerebral cortex as determined by direct recording of evoked potentials and electrical stimulation. J Neurosurg 51:476-506, 1979

50. Yaşargil MG: Microneurosurgery. Stuggart: Georg Thieme Verlag, 1994

51. Yaşargil MG, Krisht AF, Ture U, Al-Mefty O, Yaşargil D: Microsurgery of insular gliomas: part II: opening of the sylvian fissure. Contemporary Neurosurg 24(12):1-5, 2002

52. Yaşargil MG, Krisht AF, Ture U, Al-Mefty O, Yaşargil D: Microsurgery of insular gliomas: part III: pathophysiology and clinical presentation. Contemporary Neurosurg 24(13):1-5, 2002

53. Yaşargil MG, von Ammon K, Cavazos E, Doczi T, Reeves JD, Roth P: Tumours of the limbic and paralimbic systems. Acta Neurochir (Wien) 118:40-52, 1992

54. Yingling CD, Ojemann S, Dodson B, Harrington MJ, Berger MS: Identification of motor pathways during tumor surgery facilitated by multichannel electromyographic recording. J Neurosurg 91:922-927, 1999

55. Zentner J, Meyer B, Stangl A, Schramm J: Intrinsic tumors of the insula: a prospective surgical study of 30 patients. J Neurosurg 85:263-271, 1996

Manuscript submitted October 15, 2012.

Accepted December 17, 2012.

Please include this information when citing this paper: DOI: 10.3171/2012.12.FOCUS12342.

Supplemental online information:

Video 1: http://mfile.akamai.com/21490/wmv/digitalwbc.download. akamai.com/21492/wm.digitalsource-na-regional/Focus12-342Video-1.asx (Media Player).

http://mfile.akamai.com/21488/mov/digitalwbc.download.akamai. com/21492/qt.digitalsource-global/Focus_12-342-Video-1.mov (Quicktime).

Video 2: http://mfile.akamai.com/21490/wmv/digitalwbc.download. akamai.com/21492/wm.digitalsource-na-regional/Focus12-342Video-2.asx (Media Player).

http://mfile.akamai.com/21488/mov/digitalwbc.download.akamai. com/21492/qt.digitalsource-global/Focus_12-342-Video-2.mov (Quicktime).

Address correspondence to: Aaron A. Cohen-Gadol, M.D., M.Sc., Goodman Campbell Brain and Spine, Department of Neurological Surgery, Indiana University School of Medicine, 355 West 16th Street, Suite 5100, Indianapolis, Indiana 46202. email: acohenmd@gmail.com. 